\title{
CRYOGENICS FOR THE SUPERCONDUCTING MODULE TEST FACILITY
}

\author{
A. L. Klebaner and J. C. Theilacker \\ Fermi National Accelerator Laboratory \\ Batavia, IL, 60510, USA
}

\begin{abstract}
A group of laboratories and universities, with Fermilab taking the lead, are constructing a superconducting cryomodule test facility (SMTF) in the Meson Detector Building (MDB) area at Fermilab. The facility will be used for testing and validating designs for both pulsed and CW systems. A multi phase approach is taken to construct the facility. For the initial phase of the project, cryogens for a single cavity cryomodule will be supplied from the existing Cryogenic Test Facility (CTF) that houses three Tevatron satellite refrigerators. The cooling capacity available for cryomodule testing at MDB results from the liquefaction capacity of the $\mathrm{CTF}$ cryogenic system. A cryogenic distribution system to supply cryogens from CTF to MDB is under construction. This paper describes plans, status and challenges of the initial phase of the SMTF cryogenic system.
\end{abstract}

KEYWORDS: Test Facility, Superconducting, International Linear Collider

PACS: 07.20.Mc

\section{INTRODUCTION}

A group of laboratories and universities are constructing a superconducting radiofrequency (SRF) accelerator facility at Fermilab. The facility will provide the primary development and testing forum for major SRF based projects in high energy physics, most notably the International Linear Collider (ILC), Fermilab Proton Driver (PD) and Northern Illinois Center for Accelerator and Detector Development (NICADD) Photo-injector laboratory, as well as compliment the existing and planned SRF facilities at other laboratories for nuclear physics, materials, and life sciences.

The SRF test facility will be constructed in phases beginning in 2005 and proceeding through the end of the decade. These phases correspond to the expansion of the testing capabilities and SRF development at Fermilab. The initial phase plans have included the 
clean-up of the Meson Detector Building (MDB) and the establishment of the test areas, each with appropriate shielding, cryogenic capability, and AC power for RF sources.

As work on the program goals has progressed, the need to develop infrastructure for ILC related tasks and PD in parallel has become apparent. It also became clear that MDB is not suitable to support the needs of both projects simultaneously. The decision was made to split the facility between two buildings - MDB and New Muon Lab (NML). Currently, the MDB test area is dedicated to PD activities, where ILC related studies are to be done at NML.

For the ILC program in the NML, the goal is to establish a high gradient, $1.3 \mathrm{GHz}$ cryomodule test area at Fermilab with a high quality pulsed electron beam, using an upgraded Fermilab/NICADD Photo-Injector Laboratory (FNPL).

For the PD program in the MDB, the goal is to establish an area for high power, 325 $\mathrm{MHz}$, SRF testing of $\beta<1$ accelerator structures in pulsed mode ( $\sim 1 \%$ duty factor). Additionally, the MDB will house two single module test areas for cavity development programs.

\section{CRYOGENIC REQUIREMENTS}

\section{Heat Load}

The present plan at NML is to reconfigure the injector to include: a normal conducting gun, two Capture Cavities [1] operating at an average accelerating gradient of $12.5 \mathrm{MV} / \mathrm{m}$ and $30 \mathrm{MV} / \mathrm{m}$ respectively, and a $3.9 \mathrm{GHz}$ cavity. A further step will incorporate a $3.9 \mathrm{GHz}$ cavity ( $3^{\text {rd }}$ Harmonic) operating in deceleration mode. It is desirable to operate the ILC cryomodules with a gradient of $35 \mathrm{MV} / \mathrm{m}$ in the SMTF. This adds considerably to the dynamic heat load. Heat load requirements for different phases of the SMTF at NML are presented in TABLE 1 . Components that are included in each phase are marked by symbol ' $\mathrm{X}$ '. Cavity radio frequency (RF) loads were scaled from TESLA TDR [2] by the cavity Q, repletion rate (Rep rate), RF pulse length and square of gradient ratios. TESLA Test Facility (TTF) and FNPL operating experience are used for the static heat load estimates.

MDB is currently being configured to have three (3) separate test areas: PD front end, Horizontal Elliptical Cavity, and Spoke Resonator Cavity test cryostats. The PD front end consists of twenty-five (25) superconducting solenoids of 5 Tesla each, one (1) Single Spoke Resonator cryomodule, two (2) Double Spoke Resonator cryomodules, and appropriate solenoids.

TABLE 1. NML Heat Load Requirements.

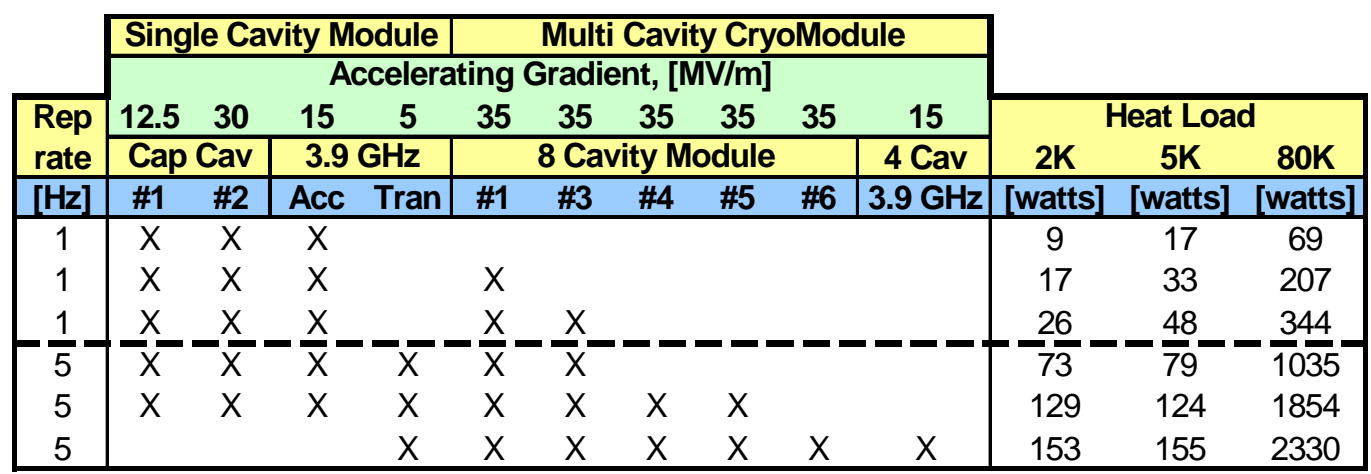


Tevatron Satellite Refrigerator Capacity Chart

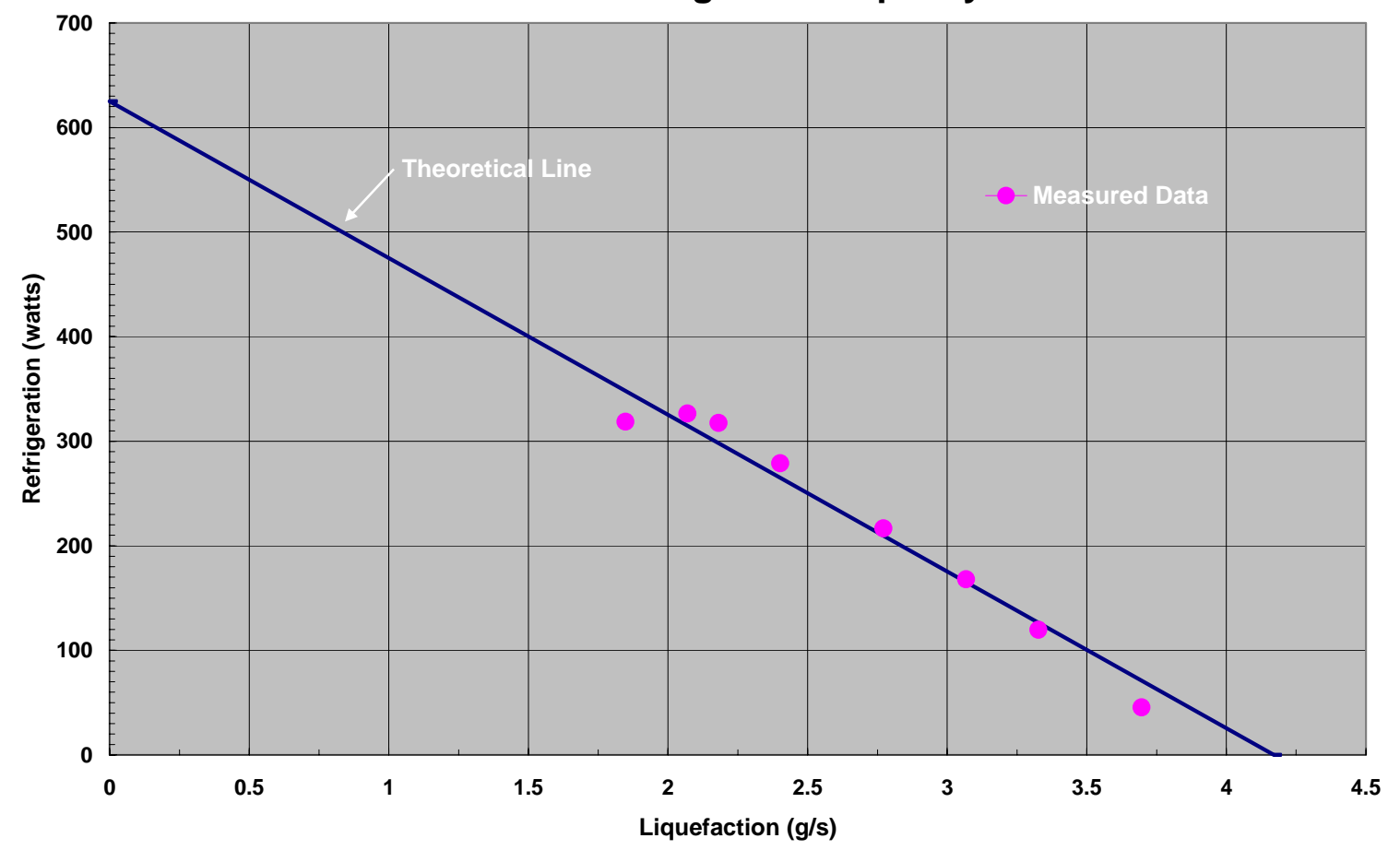

FIGURE 1. Refrigeration versus liquefaction capacity chart for a typical Tevatron Satellite refrigerator.

\section{Cryogenic Capacity}

An ambient temperature pumping cycle was chosen to provide super-fluid helium for the initial stage of the SMTF cryogenic system. Existing Tevatron Satellite refrigerators running in a liquefier mode in conjunction with existing vacuum pump systems are centerpieces of the SMTF cryogenic system.

The Tevatron satellite refrigerators are originally designed to provide $625 \mathrm{~W}$ at $4.5 \mathrm{~K}$ in

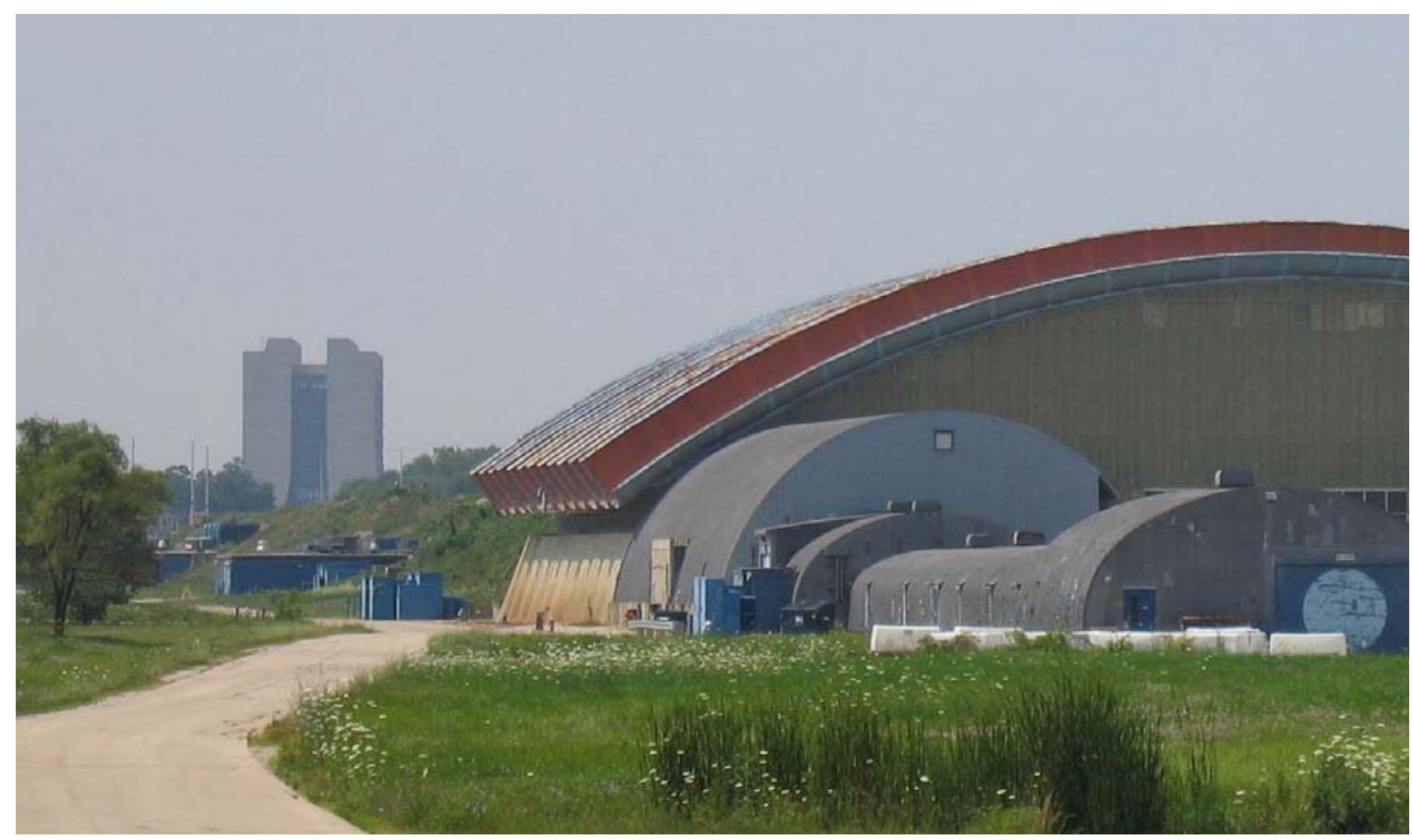

FIGURE 2. Meson Detector Building. 


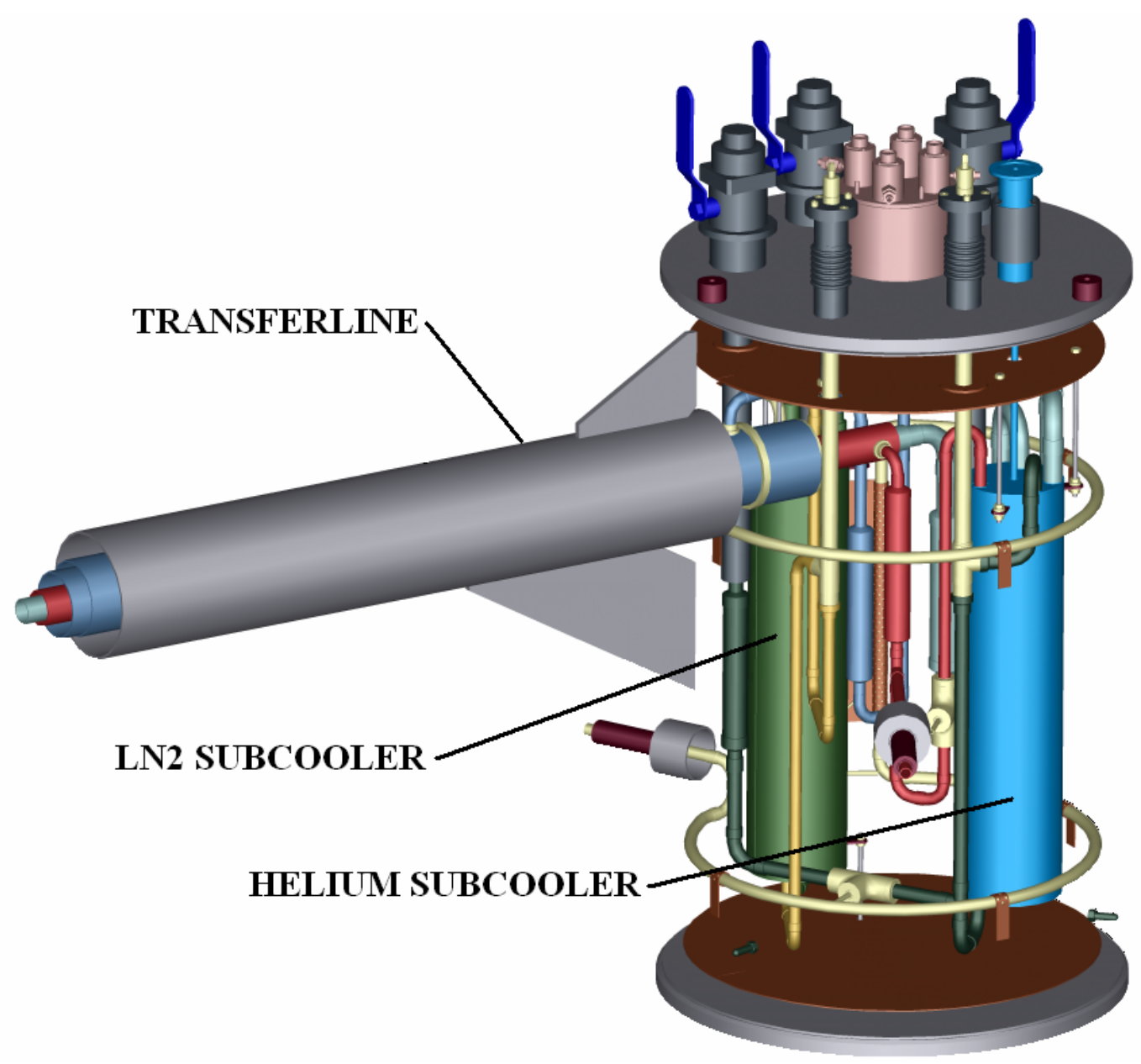

FIGURE 3. Meson Detector Building Bayonet Can designed by SLAC.

a refrigerator mode [3]. In order to determine a satellite refrigerator capacity in a liquefier mode, a series of capacity tests were conducted. The results of the tests are presented on FIGURE 1.

The two stage vacuum pump system is comprised of Kinney Model KMBD - 10,000 roots blower and Kinney Model KLRC 2100 liquid ring pump. The vacuum pump skids were assembled by our colleagues at TJNAF and are currently undergoing modification for the helium service.

\section{MESON DETECTOR AREA}

\section{Cryogenic Test Facility}

Fermilab Cryogenic Test Facility (CTF), formerly the Meson Central Cryogenics (MCC), is located on the west side of the Meson beamline, about $500 \mathrm{~m}$ southwest of the MDB. The CTF houses three (3) Tevatron satellite refrigerators capable of producing a total of $1.8 \mathrm{~kW}$ at $4.5 \mathrm{~K}$.

Part of the $4.5 \mathrm{~K}$ stream will be used in the production of superfluid helium. The remainder of the $4.5 \mathrm{~K}$ flow will be used to satisfy $5-8 \mathrm{~K}$ heat shield loads in the cryomodules and to maintain stability in the $\mathrm{CTF} / \mathrm{MDB}$ cryogenic transfer line. For convenience, the $40-80 \mathrm{~K}$ shield loads in the cryomodules will be cooled using $80 \mathrm{~K} \mathrm{LN}_{2}$. 
The CTF refrigerators will be operating in a liquefier mode. Operation of the satellite refrigerator in this mode is inherently less reliable. It is more demanding on the reciprocating expanders and heat exchanger performance. Helium impurity degrades the expander and heat exchanger performance, and thus is detrimental to the reliability and capacity of the system.

To improve reliability of the CTF refrigerators, a purifier compressor was integrated into the existing compressor system. A liquefaction porting of the main flow is continuously pumped by the purifier compressor via an in-line helium purifier prior to being returned to the refrigerators. Compressor oil removal and the piping system were modified to enable such a feature. The new piping arrangement allows for uninterrupted operation via a mobile purifier while the main in-line purifier is being regenerated.

\section{Transfer Line}

The existing transfer line and associated headers from CTF were extended to enable delivery of cryogens in the MDB. An appropriate expansion box was installed. Two new bayonet cans at each side of the transfer line are installed. The MDB side bayonet can presented in FIGURE 3 was designed by our colleagues at SLAC, while the CTF side bayonet can was designed at Fermilab. The bayonet cans were constructed at Fermilab.

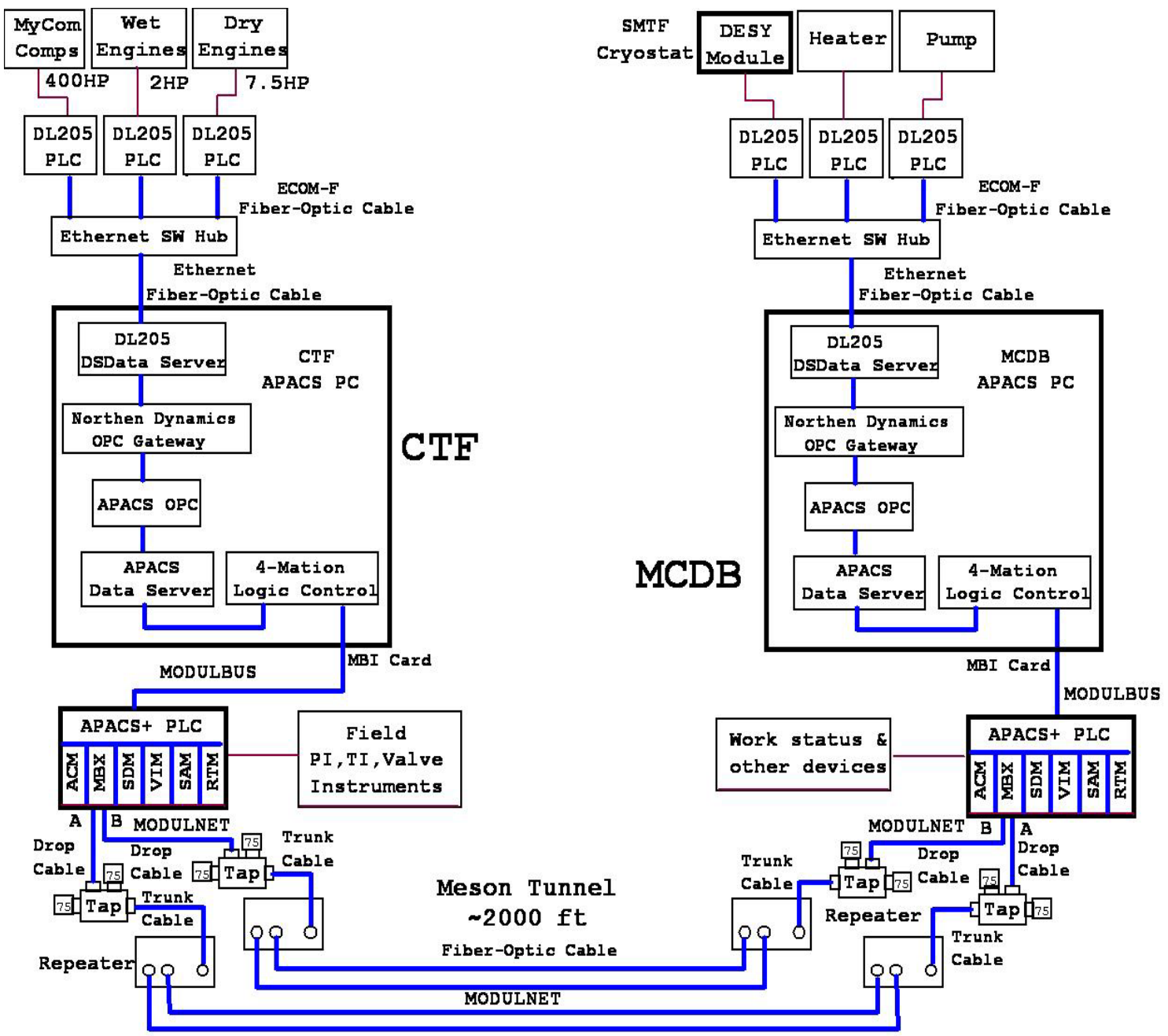

FIGURE 4. MDB Cryogenic Controls Diagram. 


\section{Vacuum Pump System}

It is imperative that the subatmospheric portion of the system remains leak tight for reliable cryogenic operation. For this, the vacuum pump system is being modified to minimize air inleaks. It is common for vacuum pumps designed for air service during stop condition to leak air in via the shaft dynamic seal. In order to eliminate these kinds of leaks, guard buffers filled with helium are being installed around dynamic seals of the pumps. A varity of valves and piping connections are being upgraded for a subatmospheric service. A variable speed drive is being installed for the roots blower.

\section{Cryogenic Controls}

A new local distributed cryogenic controls system node has been installed at MDB. The controls are based on SIEMENS-MOORE APACS $+{ }^{\circledR}$ industrial process automation system. The same brand system has been successfully used for almost a decade to control CTF. Two nodes, CTF and MDB, are networked via SIEMENS MODULNET ${ }^{\circledR}$.

A controls diagram for MDB cryogenic system is presented in FIGURE 4. For local machine control (expanders, compressor, etc.) $\mathrm{KOYO}^{\circledR}$ program logic controllers (PLC) Model DL 205 are used. A local machine PLC communicates to APACS $+{ }^{\circledR}$ controller via DSDATA $^{\circledR}$ OPC server through Northern Dynamics ${ }^{\circledR}$ OPC gateway. The Experimental Physics and Industrial Control System (EPICS) Input Output Controller (IOC) was installed to enable use of the existing set of software tools, libraries and applications developed collaboratively and used worldwide to create distributed soft real-time control

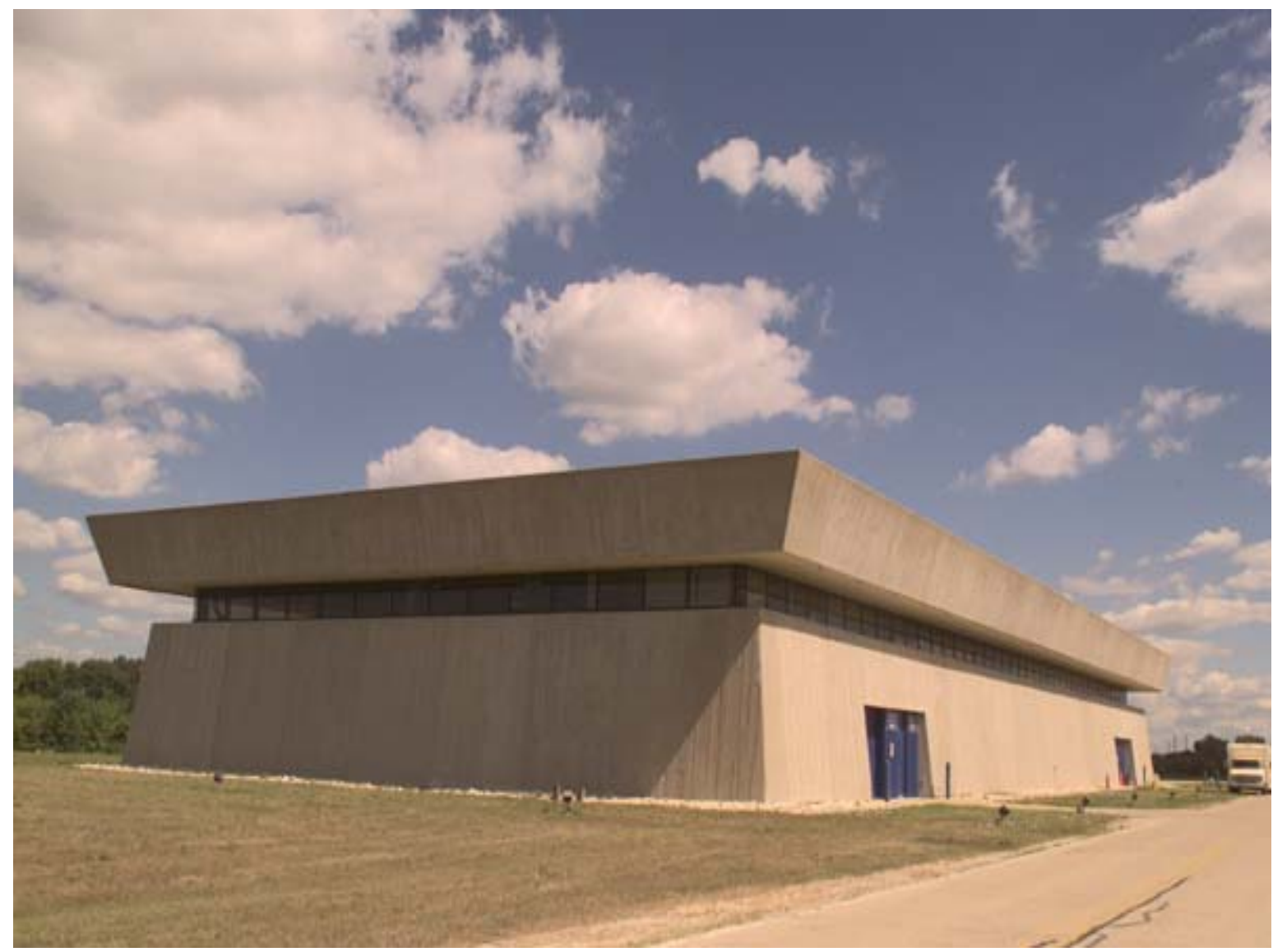

FIGURE 4. New Muon Lab Building. 
systems for the SMTF. A Data Acquisition Engine was written by Fermilab Accelerator Division Controls Department to allow data exchange and the use of applications written within the Fermilab ACNET control system.

\section{NEW MUON LAB AREA}

NML shown in FIGURE 3 is being configured to accommodate the FNPL injector and Continues Wave (CW) SRF module test area. The initial configuration of the FNPL injector includes a normal conducting gun, Capture Cavity I, Capture Cavity II, and 3.9GHz cavity. Within the next two (2) years, the injector will be upgraded with two (2) $1.3 \mathrm{GHz}$ eight cavity cryomodules. One of these cryomodules will contain cavities made in the United States and Japan, and will be assembled at FNAL. The other will be obtained from DESY. Eventually within the next five (5) years, a total of four (4) cryomodules would be installed and used to accelerate ILC-like beams.

To support the cryogenic needs of NML, a staged approach is taken. For the first stage, a temporary cryogenic system will be used. Similar to the MDB cryogenic system, the temporary NML system is based on the existing Tevatron Satellite refrigerators and warm vacuum pumps.

For the second stage, a permanent system will be installed. Investigation of the best way to proceed with a larger capacity permanent cryogenic system to support the second stage NML needs is being pursued. Two options are currently being considered: a) Superconducting Super Collider (SSC) Magnet Test Lab (MTL) plant combined with warm vacuum pumps, and b) a new hybrid cryogenic system.

\section{CONCLUSIONS}

An international panel has recommended adoption of SRF technology to accelerate the electron and positron beams of the International Linear Collider. Nearly all new initiatives for electron, ion and proton sources incorporate superconducting cavities. These projects place increasing demands on performance of SRF, and the ability to produce SRF components in large quantity at lower cost.

The SMTF will allow collaborators to benefit from the oversight and resources of Fermilab, will avoid needless and costly duplication of effort, and will create a scientific synergy that will assure success. The R\&D performed at SMTF will be of value to several planned accelerator projects, including ILC and PD.

\section{ACKNOWLEDGEMENTS}

The authors wish to express their appreciation and thanks to all those at Fermilab, SLAC and TJNAF for their dedicated effort, hard work and contributions to the SMTF project. Fermilab is operated by Universities Research Association Inc. under Contract No. DE-AC02-76CH03000 with the United States Department of Energy.

\section{REFERENCES}

1. Buhler, S. et al, "Status Report of the TTF Capture Cavity Cryostat," in Advances in Cryogenic Engineering 41, edited by P. Kittel, Plenum, New York, 1996, pp. 863-867. 
2. Andruszkow, J. et al., TESLA Technical Design Report part II, edited by R. Brinkmann, DESY, Hamburg, 2001, p. 81.

3. Cole, T. F. et al., "A Report on the Design of the Fermi National Accelerator Laboratory Superconducting Accelerator," FERMILAB, Batavia, 1979, p. 71. 